


\title{
ACERCA DE LA PSICOLOGÍA DE LA RELIGIÓN Y LA ESPIRITUALIDAD
}

\author{
Cecilia Salgado Lévano*
}

El presente trabajo tiene como objetivo ofrecer una revisión sistemática de lo que comprende la Psicología de la religión y espiritualidad, para ello en primer lugar se revisan las definiciones, condiciones y dimensiones de la religión, en segundo lugar, las definiciones y dimensiones de la religiosidad y en tercer lugar, las definiciones, modelos en salud, dimensiones y características de la espiritualidad. Se concluye que a pesar de los avances en este campo, aún es necesario destinar esfuerzos personales y colectivos para impulsar investigaciones, dado el innegable rol que tiene en la vida del ser humano variables como el afrontamiento religioso, la convicción espiritual, el bienestar espiritual, las experiencias y formas de participación religiosa, creencias y prácticas espirituales y religiosas, la conversión y la fe en Dios.

Palabras Claves: Espiritualidad, Psicología de la Religión y Espiritualidad, Religión, Religiosidad.

This paper aims to provide a systematic review of what comprises the psychology of religion and spirituality, for this first definitions, conditions and dimensions of religion, secondly, definitions and dimensions of religiosity are reviewed and thirdly, definitions, health models, dimensions and characteristics of spirituality. It is concluded that despite advances in this field, it is still necessary to allocate personal and collective efforts to promote research, given the undeniable role that the life of the human variables such as religious coping, spiritual conviction, spiritual, experiences and forms of religious participation, and spiritual beliefs and religious practices, conversion and faith in God.

Keywords: Spirituality, Psychology of Religion and Spirituality, Religion, Religiosity.

Doctora en Psicología (Universidad Nacional Mayor de San Marcos); Máster universitario en Ciencias para la familia (Universidad de Málaga); Maestra en Ciencias con mención en Psicología (Universidad Peruana Cayetano Heredia); Docente de postgrado (Universidad Marcelino Champagnat); Docente de pregrado (Universidad San Ignacio de Loyola); Premio Nacional de Investigación 2002 otorgado por el Colegio de Psicólogos del Perú; e-mail: csalgadolevano@gmail.com

I. Forma parte de la tesis doctoral de la autora. 


\section{Introducción}

El fenómeno religioso ha estado presente desde tiempos inmemoriales en la humanidad, forma parte de la especie humana y no sólo es potestad de la Psicología, sino de las demás disciplinas ligadas al hombre. Al respecto, García-Alandete y Pérez (2005) afirman que el fenómeno religioso, en sus diversas manifestaciones, tanto subjetivas (personales) como objetivas (colectivas), siempre ha estado y sigue estando, en el punto de mira de diferentes disciplinas humanas y sociales, tales como la Filosofía, la Sociología y la Psicología, configurando ámbitos de investigación específicos.

Es necesario recordar que entre los intereses iniciales de la Psicología se encontraba la espiritualidad (Pargament \& Mahoney, 2002; Spilka, 2003). Sin embargo, posteriormente fue dejada de lado e ignorada por los psicólogos, que la concebían como algo patológico o como un proceso que podía reducirse a funciones psicológicas, sociales y biológicas subyacentes más básicas (Pargament \& Mahoney, como se citó en San Martin, 2007).

No cabe duda que durante décadas, se ha evitado investigar la espiritualidad por considerársele nada científico. Al respecto Rivera (2007) señala que el área espiritual de la vida humana ha sido largamente segregada del campo de la psicología, y parece ser necesario, hoy por hoy, asignarle un lugar más allá del apasionamiento de la fe y el escepticismo academicista contemporáneo.

En esta línea, se constituyó inicialmente la "Psicología de la Religión", entendida como la rama de la psicología aplicada y de la ciencia de la religión, que abarca las manifestaciones psicológicas vinculadas a la práctica religiosa, cuyo centro de estudio son las creencias, actividades y experiencias religiosas desde el punto de vista psicológico (Font, como se citó en Quiceno y Vinaccia, 2009).

Sin embargo, dicha denominación como rama de la psicología cambio, debido a que a mediados del siglo $X X$ comenzaron a tener auge investigaciones relacionadas con la espiritualidad, la religión y la salud (González, 2004; Hill et al., 2000). Más concretamente, en las últimas décadas se han publicado investigaciones en revistas médicas y psicológicas, en las cuales Harold G. Koenig, Kenneth I. Pargament, Pamela G. Reed, David B. Larson y Jeffrey S. Levin, aparecen como algunos de los pioneros que abrieron una nueva etapa para la investigación científica de la religión y la espiritualidad en el área de la salud (Moreira-Almeida, Lotufo \& Koenig, 2006). Lo que posibilitó que se incorporara académicamente el estudio de la espiritualidad, con lo cual paso de ser solamente "Psicología de la Religión" a llamársele "Psicología de la Religión y la Espiritualidad", que hace referencia a las experiencias y formas de participación religiosa, creencias y prácticas espirituales-religiosas, el afrontamiento religioso, la conversión y la fe (Yoffe, como se citó en Quiceno y Vinaccia, 2009). 
La denominación de "Psicología de la Religión y la Espiritualidad", es mucho más amplia y rica, dado que como se sabe la religión hace referencia a una serie de prácticas y normas acorde a las creencias que se tiene; no obstante, el sustrato a todas ellas es la espiritualidad, entendiéndola como se verá más adelante, como aquella que precisamente nutre la religión y le da sentido, es decir, la fe en Dios.

A continuación se hará una revisión sobre la religión, la religiosidad y la espiritualidad, dado que son conceptos medulares al abordar la Psicología de la Religión y Espiritualidad.

\section{CONCEPTUALIZACIONES SOBRE LA RELIGIÓN}

Es necesario que se conozcan algunas conceptualizaciones acerca de la religión, dada su íntima relación con la espiritualidad.

\section{Definiciones}

Solimine y Hoemman (como se citó en Pinto, 2007) describen la religión como un sistema organizado de adoración, en el que la creencia y las normas morales son mantenidas en un ritual formal y común, al igual que tiene una serie de observaciones, producto de la práctica que pueden ser una expresión de la espiritualidad de una persona, pero no su núcleo.

La religión según Jiménez (2005) es definida como la creencia en la existencia de un poder reinante sobrenatural, creador y controlador del universo, que ha dado al hombre una naturaleza espiritual que continúa existiendo después de la muerte del cuerpo.

Para Cabestrero (como se citó en Rodríguez, Fernández, Pérez y Noriega, 20 I I) la religión puede ser entendida como el conjunto de instituciones que formulan, organizan, administran o coordinan el instrumental de teorías, doctrinas, dogmas, preceptos, normas, signos, ritos, símbolos, celebraciones o devociones, en torno a una creencia trascendental y a través de las cuales se conservan, cultivan o expresan colectivamente las experiencias espirituales personales.

Pinto (2007) considera que la religión alude a una institución cultural u organización grupal, en torno a un culto específico, que tiene lugar y tiempo particulares, ofrece consuelo en las privaciones, favorece la autoaceptación y disminuye los sentimientos de culpa.

La religión sitúa la vida espiritual en una comunidad con una tradición. Ella se basa en una fe en Dios, busca una organización y una expresión comunitarias así como un apoyo para la vida espiritual (Vanistendael, 2003). 
Desde la persona como parte de un sistema cultural, la religión sirve como un lente para leer el mundo, el yo y las relaciones que se establecen entre ellos (Korman, Garay y Sarudiansky, 2008). Es decir, orienta la percepción y permite ver la realidad de una manera más rica y profunda.

Como se puede apreciar existen algunas diferencias entre las definiciones planteadas por los autores, aunque en todas ellas, hay un hilo conductor, entendido como el conjunto de prácticas, creencias, rituales, símbolos que se viven a nivel institucional y que tienen como base la relación con Dios, el modo como se le percibe y qué actitudes se tienen frente a Él, de ahí dependerá la asunción del estilo religioso que se viva, basado solo en lo superficial, entendido como el mero cumplimiento de normas y prácticas propias de una religión, o como la manifestación coherente de una relación profunda de amor a Dios, que se alimenta por el amor que proviene de Él y la fe que suscita.

\section{Condiciones personales y sociales}

Es evidente que el concepto integral del hombre; supone que el hombre no es tan sólo un ser bio-psico-social, sino también espiritual y religioso (Jaramillo, Carvajal, Marín y Ramírez, 2008). Prescindir de esta dimensión de la vida del ser humano sería desconocer aspectos medulares del desarrollo de la persona.

De acuerdo a Rivera (2007) el concepto religión es de naturaleza social; se construye a partir de la coincidencia en muchos hombres y mujeres de un lugar y una época dada, de dos situaciones eminentemente humanas e individuales; por un lado, la experiencia de lo espiritual, y por otro, la ansiedad existencial que plantea el sólo hecho de vivir y la conciencia de ser finito. La religión constituye el campo social al que a menudo se atribuye y donde a menudo se expresa la vida espiritual.

Es importante analizar el desarrollo religioso del ser humano, el cual implica la aceptación de determinadas creencias, valores, normas de conducta o rituales. Puede o no ir paralelo al desarrollo espiritual. Por ejemplo, alguien puede seguir ciertas prácticas religiosas, pero no interiorizar el significado simbólico que hay detrás de las prácticas (Goldberg, como se citó en Pinto, 2007). O alguien puede considerarse una persona muy espiritual, pero no seguir ninguna religión en particular, sino creer en Dios a su manera (aunque esto puede resultar polémico).

Es necesario entonces tener en cuenta por un lado, las características personales del creyente y por otro lado, las características sociales del entorno. Pueden haber personas, y de hecho las hay que utilizan la religión para ganar status, para encontrar compañía, para intentar superar sus odios, resentimientos y/o sentimientos de culpa, dejando de lado, la base de toda práctica religiosa, como es la espiritualidad que precisamente nos mueve a ella, nos mueve a Dios, permitiéndonos reconocerlo 
como autor de la vida. Por tanto, toda práctica religiosa desprovista del sostén que da la verdadera fe en Dios, se convertirá solo en un conjunto de prácticas ajenas a su esencia más importante, como es el amor a Dios.

Asimismo, es necesario conocer y entender las características sociales del entorno donde se desarrolla la religión, para discernir cuando hay patología y cuando no la hay. Como bien señalan Korman et al. (2008) desde la perspectiva de los profesionales de la salud mental, muchas prácticas religiosas pueden ser consideradas patológicas, si no es tenido en cuenta el contexto. Debe recordarse que si bien es cierto, la religión es entendida como un sistema de creencias y prácticas, éstas son vividas, compartidas, sentidas por un grupo de personas que según Johnstone (como se citó en Rodríguez, 2007) interpretan y responden a lo que ellos sienten que es sagrado y sobrenatural.

En este sentido la religión aunque tiene un correlato individual, es un fenómeno grupal y por lo tanto es una forma de interacción y de comunicaciones entre grupos de personas, hay metas y normas compartidas, hay roles, funciona colectivamente mediante un sistema de status, y hay un sentido de identificación con el grupo. Sin embargo, no se trata de un grupo cualquiera, sino que está concentrado en lo sobrenatural o lo sagrado, sobre lo cual desarrolla un cuerpo de creencias, un conjunto de prácticas e involucra una serie de prescripciones morales (Rodríguez, 2007).

\section{Dimensiones}

Beit-Hallahmi y Argyle (como se citó en Quiceno y Vinaccia, 2009) proponen la consideración de la religión como una actitud compuesta de tres dimensiones: una cognitiva (creencias religiosas), comportamental (comportamientos religiosos y rituales más o menos institucionalizados y convencionales) y afectiva (vínculos entre el hombre y la transcendencia) que nosotros particularmente creemos que es más pertinente llamar "Dios".

Por otro lado, es claro que la religión contiene numerosas variables que no siempre tienen relación entre sí y por lo tanto no se puede considerar la religión como un aspecto unidimensional de la existencia humana, sino como algo multidimensional (Flannelly, Weaver \& Costa, 2004; Kendler, Gardner \& Prescott, 1997; King \& Dein, 1998; Larson et al., 1992). Por ejemplo, sería importante considerar por separado los aspectos relacionados con el apoyo social de los aspectos relacionados con las experiencias individuales de las personas (King \& Dein, 1998) dentro de las que estarían según Larson et al., (1992) variables relacionadas con el sentido, tipo y frecuencia de práctica religiosa y sobre cómo se vive la relación con Dios (como se citó en Rodríguez, 2006). 


\section{CONCEPTUALIZACIONES SOBRE LA RELIGIOSIDAD}

\section{Definiciones}

Para Emmons y Paloutzian (como se citó en Rodríguez, 2006) la religiosidad es la forma que cada uno utiliza para expresar sus creencias religiosas y la importancia que les confiere dentro de un contexto cultural determinado. Puede considerarse como el conjunto de prácticas que realizan los que profesan una religión (oración, lectura de textos sagrados, rituales, etc.). También se puede definir la religiosidad como una característica personal que tiene que ver con un sistema de creencias y que proporciona sentido que es estable en el tiempo y que se pone de manifiesto en diferentes situaciones.

Para jiménez (2005) la religiosidad es el grado en el que una persona cree, sigue y practica una religión. En este caso hay un sistema de culto y una doctrina específica que es compartida por un grupo.

Otros consideran que la religiosidad es la expresión comportamental del sistema de creencias, la doctrina y los cultos organizados de la religión. La religiosidad se vive en lo social como un cuerpo de conocimientos, comportamientos, ritos, normas y valores que rigen o pretenden regir, la vida de personas interesadas en vincularse con lo divino. Pretende ser un compromiso (no siempre logrado) con creencias y prácticas características de una tradición religiosa particular (Peteet, 1994), expresada según Walker y Pitts (1998) en credos y rituales (como se citó en Rivera, 2007).

Según Milanesi y Aletti (como se citó en Gallego-Pérez, García-Alandete y PérezDelgado, 2007) la religiosidad se enmarca dentro de la tentativa de "dar un significado" al hombre, al mundo y a la relación entre ambos, de modo que la experiencia religiosa se halla íntimamente vinculada al fenómeno humano de la búsqueda de significado de la realidad, en su sentido más amplio, y, de manera especial, de la existencia personal. Ofrece un horizonte trascendente de sentido desde el cual orientar la propia existencia. Se inserta, con ello, en un proceso de búsqueda global de sentido.

Es evidente entonces la relación que existe entre religión y religiosidad, la religión es el conjunto de normas, rituales y prácticas vividas como parte de un sistema institucionalizado; mientras que la religiosidad es precisamente la expresión comportamental de la misma. En este sentido, nos podemos preguntar iCómo vive cada persona esas normas o prácticas que profesa según su religión?, ilas vive a conciencia?, ide modo coherente o incoherente?, ipleno de convencimiento en lo que profesa o solo lo sigue por seguir, porque forma parte de sus tradiciones o una cultura determinada? 


\section{Dimensiones}

Allport y Ross (como se citó en Rodríguez, 2006) diferencian entre religiosidad intrínseca y extrínseca. La religiosidad intrínseca es una expresión integrada de los pensamientos y prácticas religiosas. Las personas con religiosidad intrínseca, se toman la religión muy en serio, la asumen plenamente en su vida cotidiana y encuentran en la religión el fundamento de sus motivaciones.

De hecho que la religiosidad intrínseca estaría totalmente vinculada a la espiritualidad -como se verá más adelante-. Al respecto algunos autores, como por ejemplo, Fehring, Miller y Shaw (como se citó en Martínez, Méndez y Ballesteros, 2004) consideran que la religiosidad intrínseca, concierne a la espiritualidad como tal. Probablemente esta sea una de las bases para entender por qué muchas veces se superponen ambos conceptos, ya que el centro de ambos es la relación con la divinidad, es decir, con la manera como la persona se relaciona con Dios, aspecto medular de la espiritualidad.

Una manifestación particular de la religiosidad intrínseca es la oración, que ha pasado a constituirse en uno de los más significativos por la influencia positiva que ejerce en el mantenimiento y restitución de la salud, o en la prolongación de la vida de quienes la emplean (Helm, Hays, Flint, Koenig \& Blazer, 200 I; Mackenzie, Rjagopal, Meibohm \& Lavizzo-Mourey, 2000; VandeCreek, Pargament, Belavich, Cowell \& Friedel, 1999. Como se citó en González, 2004).

En los últimos años el interés por el estudio de la oración se ha ampliado, al extenderse el dominio de la oración no sólo hacia la propia persona que la realiza, sino, hacia el influjo que ejerce a distancia sobre otras personas, particularmente la oración intercesora, ha pasado a constituir dentro del contenido religión-salud uno de los de mayor consideración (González, 2004), lo cual se ve reflejado en diversas investigaciones que se realizan.

Por otro lado, la religiosidad extrínseca se refiere a las prácticas rituales y a las religiones institucionales (Fehring, Miller \& Shaw, como se citó en Martínez et al., 2004). Rodríguez (2006) considera que la religiosidad extrínseca, se refiere al uso de las prácticas externas de la religión para buscar relación social, seguridad o status. Es decir, se podría llegar a usar la religiosidad como un instrumento de las propias motivaciones y no como el origen de éstas.

Sin embargo, ello no necesariamente refleja la realidad, pues aunque hay personas que efectivamente pueden buscar en la práctica externa de la religión algo no relacionado a Dios, existen personas que por el contrario, a través de dichas prácticas buscan vivir de modo coherente su fe y amor a Dios, su sentido de vida y consecuentemente su capacidad de trascendencia. 
Otra manera de entender la religiosidad, es a través de cómo se expresa la relación con Dios, manifestándose en dos momentos: subjetivo y objetivo. En relación con el momento subjetivo, la religiosidad, como experiencia subjetiva e íntima, adquiere el carácter de una experiencia de encuentro (Velasco, 1976). Mientras que el momento objetivo según Milanesi y Aletti (1974), se refiere a que la religiosidad personal, en términos de experiencia religiosa, se inserta en un contexto histórico, cultural y social, experimentando un proceso de institucionalización que la canaliza de manera que pueda formar parte de una experiencia colectiva (como se citó en García Alandete, 2002).

\section{CONCEPTUALIZACIONES SOBRE LA ESPIRITUALIDAD}

La espiritualidad es un concepto que se origina en la filosofía y en la teología hace varios siglos, aunque dentro de la terminología científica es un constructo relativamente nuevo (Rodríguez et al., 20 I I). Filósofos y pensadores han llegado a la conclusión -que actualmente es compartida por los psicólogos occidentales- del importante papel que cumplen en la vida de las personas, la espiritualidad (Abi-Hashem, $200 \mathrm{I}$. Como se citó en Martínez, 2006).

La literatura documenta las diferentes formas en que las personas experimentan la espiritualidad y la admiten como una condición universal (Stoll, 1979; Burnard, 1988. Como se citó en Sánchez, 2009). El interés en este tema es indicativo de la concientización en varios sectores académicos y profesionales sobre la necesidad de considerar la espiritualidad como una parte esencial e integral del desarrollo de la personalidad del ser humano (Richard \& Bergin, 1997; Corey, 1996. Como se citó en Pérez Santiago, 2007). Es claro que el ámbito de la espiritualidad, vincula lo profundamente personal con lo universal y es esencialmente unificador (Jiménez, 2005). Según Sánchez (2009) los seres humanos son espirituales, y muchos de ellos, religiosos.

Se ha destacado que el ser humano necesita a Dios porque constituye la solución al problema de su muerte; a su incapacidad de evadir su propia muerte; la solución al miedo, a la ansiedad de vivir en el sin sentido, de vivir una vida sin propósito; es el problema de la finitud humana lo que lleva al hombre a preguntarse sobre la existencia de Dios (Tillich, 1952a, 1952b; Meadow \& Kahoe, 1984. Como se citó en Rivera, 2007).

Probablemente estas sean las razones por la cuales el ser humano es profundamente espiritual, lo cual es evidente en todas las culturas y tiempos, es decir, la espiritualidad es una dimensión esencial a la vida humana. Sin embargo, en la vida cotidiana, y a pesar de tener muchas manifestaciones de la espiritualidad, las personas no siempre son conscientes de ella. Por el contrario, la espiritualidad resulta evidente cuando se siente la vida amenazada (Reyes-Ortiz, como se citó en Sánchez, 2009) ante la presencia de enfermedades, pérdidas de diversa índole, situaciones extremas e incluso, la muerte. 


\section{Definiciones}

Según Chochinov y Cann (como se citó en Beca, 2008) se han referido más de 90 intentos de definiciones de espiritualidad, las que incluyen conceptos tan variados como la relación con Dios o un ser espiritual, algo superior a uno mismo, trascendencia, significados y fines de la vida, fuerza vital de la persona, vida interior, paz interior, comunión con otros, contacto con la naturaleza, relaciones con familiares y amigos, entre otros. A continuación, revisaremos varias definiciones que los científicos han planteado acerca de la espiritualidad.

Para Moberg (como se citó en Rodríguez et al., 20 I I) es el conjunto de creencias trascendentales sobre Dios y la relación con Él, y de creencias existenciales en cuanto a la relación consigo mismo y con los demás, que proporcionan un propósito y satisfacción a la persona.

Según el Cristianismo, la espiritualidad es un don del Espíritu Santo, un regalo de Dios: "Y nosotros no hemos recibido el espíritu del mundo, sino el Espíritu que viene de Dios, y por Él entendemos lo que Dios, en su bondad, nos concedió" ( I Corintios 2: I2. Biblia Latinoamérica).

Según Jiménez (2005) una definición de espiritualidad amplia, que puede facilitar el encuentro de bases comunes entre diversas culturas, incluye necesidades humanas que posiblemente son universales:

I. La necesidad de encontrar sentido, propósito y realización en la vida (la necesidad de darle un sentido a la vida es un rasgo universal que es esencial a la vida misma y por el contrario, la falta de sentido puede engendrar sentimientos de vacío y desesperación).

2. La necesidad de esperanza o de voluntad de vivir (la necesidad de esperanza y voluntad de vivir es importante tanto para personas sanas como personas enfermas).

3. La necesidad de creer, tener fe en uno mismo, en los otros o en Dios (contribuye a dar sentido a la vida y puede tener influencia sobre el nivel de esperanza y el deseo de vivir).

Por otro lado, la espiritualidad puede ser entendida como mística y ascética, es decir, como la huella de Dios en el alma y el esfuerzo del hombre por encontrar a Dios (Sánchez, 2007). La espiritualidad es el caminar por la vida desde la fragmentación, desarmonía y desintegración hacia la totalidad, armonía e integración en las relaciones con Dios, con uno mismo, con los demás y con la creación (Bek, como se citó en Ortiz, 2007). 
Según Pinto (2007) la espiritualidad se puede entender como un sistema de guía interna, básico para el bienestar humano, que influye en la vida, la conducta y la salud, sin importar la filosofía, las creencias o las prácticas religiosas de la persona. Con sabiduría, entendimiento, creencia y amor, la espiritualidad tiene el poder de dar forma y significado al patrón de autorrealización de una persona, expresado en el ser, el saber y el hacer de una perspectiva espiritual creativa y energética. La espiritualidad es un acto incuestionablemente vital, en tanto que su relación vital con el alma alude, en cierto modo, a la capacidad de trascendencia. Tal concepto conlleva la percepción de la espiritualidad como un proceso de interacción entre la conciencia y la interrelación con Dios o con un poder superior, en función de lo que se denomina fe.

San Martin (2007) afirma que la espiritualidad es un conjunto de sentimientos, creencias y acciones que suponen una búsqueda de lo trascendente, sagrado o divino. En tanto representaciones acerca de un poder final último, contribuyen a dar un sentido y propósito en la vida, y orientan la conducta de las personas, sus relaciones interpersonales y su forma de sentir y de pensar, respecto a la realidad como a sí mismos.

La espiritualidad se entiende como el conjunto de aspiraciones, convicciones, valores y creencias que permiten a cada persona orientar sus proyectos de vida (Beca, 2008). Para Quiceno y Vinaccia (20 I I) la espiritualidad se refiere a la búsqueda personal para entender las respuestas a las últimas preguntas sobre la vida, su significado y la relación con lo sagrado o lo transcendente, que puede (o no puede) conducir al desarrollo de rituales religiosos y la formación de una comunidad.

La vida individual o comunitaria se transforma cuando se trata de vivirla en relación con esta realidad espiritual, en el sentido amplio, o en el sentido más preciso de Dios. Se llama a este proceso de transformación "vida espiritual", o en una palabra "espiritualidad" (Vanistendael, 2003).

De las definiciones revisadas, puede notarse con claridad que hay un eje central en todas ellas, que es la relación con Dios, que precisamente genera la necesidad de trascender, de ir más allá, en armonía con uno mismo y con los demás pero siempre de cara a Dios, lo cual proporciona un propósito y satisfacción a la persona, un sentido a su vida.

A continuación se presentará una clasificación de las definiciones de espiritualidad.

\section{Clasificación de las definiciones según perspectivas}

De acuerdo a Krishnakumar y Neck, (como se citó en Pérez Santiago, 2007) las diversas definiciones en la literatura sobre el tema de la espiritualidad pueden 
agruparse en tres perspectivas: la perspectiva de origen intrínseco, la perspectiva religiosa y la perspectiva existencial.

La perspectiva de origen intrínseco argumenta que la espiritualidad es un concepto o principio que se origina en la interioridad de la persona. Esta perspectiva sostiene que la espiritualidad no se limita a las reglas de la religión y que es capaz de trascenderla. Involucra un sentimiento de estar conectado con uno mismo, los demás y el universo entero. Dado lo anterior, la espiritualidad se entiende como una búsqueda interna de significado que puede llevarla a cabo cualquier persona sin importar si pertenece o no a una denominación religiosa particular.

La perspectiva religiosa de la espiritualidad es aquella que emana de las creencias e instituciones asociadas a una religión en particular. Se apoya en la existencia de un Ser Supremo que rige los destinos de las personas, ofreciéndoles a sus seguidores reglas, rituales y prácticas sobre la convivencia y el comportamiento moral. La dimensión espiritual se realza en la medida en que la persona cumple con estas normativas institucionales.

La perspectiva existencial hace mayor énfasis en la búsqueda de significado personal en las vidas. Esta búsqueda de significado es activa y se da en todos los contextos sociales en los cuales se interactúa, tales como la comunidad, familia, pareja y trabajo. En esta perspectiva toma singular importancia la consistencia valorativa entre los principios ético-morales del individuo y las exigencias del ambiente externo. La concordancia o no entre estos dos aspectos propician el cuestionamiento continuo sobre la contribución relativa de las actividades en las que la persona se involucra a su sentido de bienestar y armonía individual.

Indudablemente, se pueden encontrar definiciones que con claridad aborden una de las perspectivas, pero también se van a encontrar definiciones que integren dos o tres perspectivas, ya que por ejemplo, podría existir la espiritualidad cuya base es religiosa, pero se asienta en un origen intrínseco y tiene implicancias a nivel existencial; es decir, se pueden encontrar definiciones que señalan que el centro de la espiritualidad es Dios, la relación que existe con Él, el amor dirigido a Él, pero vivida en la intimidad de la persona, y manifestada en las relaciones que establece la persona con los demás, lo mismo que le otorga sentido y significado a su vida.

Es decir, si bien es cierto, la sistematización de las definiciones según perspectivas puede ser útil, no se debe pensar que se van a encontrar totalmente diferenciadas unas de otras. 


\section{Modelos de espiritualidad en salud}

Koenig (como se citó en Quiceno y Vinaccia, 2009) planteó cuatro modelos sobre la relación de la salud mental y física con la religión y la espiritualidad o secularidad (laicidad), las mismas que seguidamente se describirán:

Versión tradicional-histórica de espiritualidad:

Se caracteriza por la profunda religiosidad, dedicación al servicio de la religión y los miembros de una comunidad y la enseñanza de las tradiciones de la fe a través del testimonio de vida. En esta versión, la religión, la espiritualidad y la secularidad (laicidad) son recursos que pueden promover valores morales, conexiones con otros, tranquilidad, armonía, bienestar, esperanza, rasgos positivos de carácter y estados mentales positivos como el propósito y significado de la vida.

La versión tradicional-histórica de espiritualidad puede ser estudiada usando los métodos de investigación de las ciencias sociales y conductuales. La espiritualidad es aquí distinta de las formas más superficiales o menos devotas de religión y de la secularidad (laicidad). Las personas espirituales pueden ser identificadas a través de medidas de participación religiosa que luego son comparadas con aquellas que son menos religiosas y con individuos seculares (laicos). La espiritualidad en esta versión es entonces un constructo completamente separado de las medidas de salud mental o física.

Versión moderna de espiritualidad:

Se caracteriza porque amplía o va más allá del constructo de religión tradicional. E término espiritualidad ha sido utilizado más ampliamente en la asistencia de salud, cuyo objetivo ha sido aplicarlo tanto a personas de diversos credos religiosos como aquellas que no lo tienen. Lo cual abre una nueva categoría de "personas espirituales, pero no religiosas". Esta versión conceptualiza la comparación de la salud mental y física de los que son "espirituales religiosos", los que son "espirituales, pero no religiosos", y los que son "completamente seculares".

Versión tautológica moderna de espiritualidad:

Aunque es similar a la anterior (versión moderna) se caracteriza porque se extiende hacia fuera incluyendo en su definición, la salud mental positiva y los valores humanos. Este concepto de espiritualidad incluye no sólo indicadores religiosos tradicionales o una búsqueda de lo sagrado, sino también estados psicológicos positivos como propósito y significado de la vida, la conexión con los demás (la calidad de apoyo social), tranquilidad, armonía y bienestar. Se define como versión "tautológica" porque incluye indicadores de salud mental en la definición de espiritualidad, lo que asegura una correlación positiva entre estas dos variables. 
Versión clínica moderna de espiritualidad:

Esta versión se caracteriza porque no solo incluye las anteriores, es decir, el constructo de religión y los indicadores positivos de salud mental, sino también lo secular (laicismo) como elementos de su definición. En este modelo es considerado espiritual incluso lo agnóstico y lo ateo.

Como se puede apreciar estos modelos pueden ser útiles para clarificar las relaciones entre la espiritualidad y la salud mental.

\section{Dimensiones de la espiritualidad}

Stoll (como se citó en Pinto, 2007) describe la espiritualidad como un concepto bidimensional que tiene una dimensión vertical y una dimensión horizontal, en permanente interrelación.

Por su parte, Montero y Sierra ( 1996 ) basándose en los aportes de Moberg y Brusek recogidos en Paloutzian y Ellison señalan que la dimensión vertical alude al bienestar derivado de la relación con Dios; mientras que la dimensión horizontal se refiere a la percepción que posee la persona sobre el propósito y satisfacción con la vida, al margen de cualquier adhesión a algún grupo religioso en particular.

Para Sánchez (2004) la trascendencia de la espiritualidad es asumida en estos dos planos. A la dimensión vertical, le añade además de la relación con Dios, la relación con un ser superior, una fuerza superior o los valores supremos. Mientras que la dimensión horizontal, es la relación de la persona consigo misma y su relación con los demás, el contexto y la naturaleza. En esta línea, el bienestar espiritual refleja una armonía entre los diferentes significados y niveles de trascendencia.

Es decir, el bienestar espiritual, puede ser entendido como la armonía entre la dimensión vertical y la dimensión horizontal de la espiritualidad, en la medida que sean coherentes entre sí. Pero la base para que esta vinculación sea coherente y efectiva, será la dimensión vertical, es decir, la relación que se establece con Dios, por ejemplo, no se podría decir que alguien alcanza el bienestar espiritual, amando a Dios pero odiando a los demás.

A continuación, se presenta la figura I que permite visualizar las dimensiones de la espiritualidad 


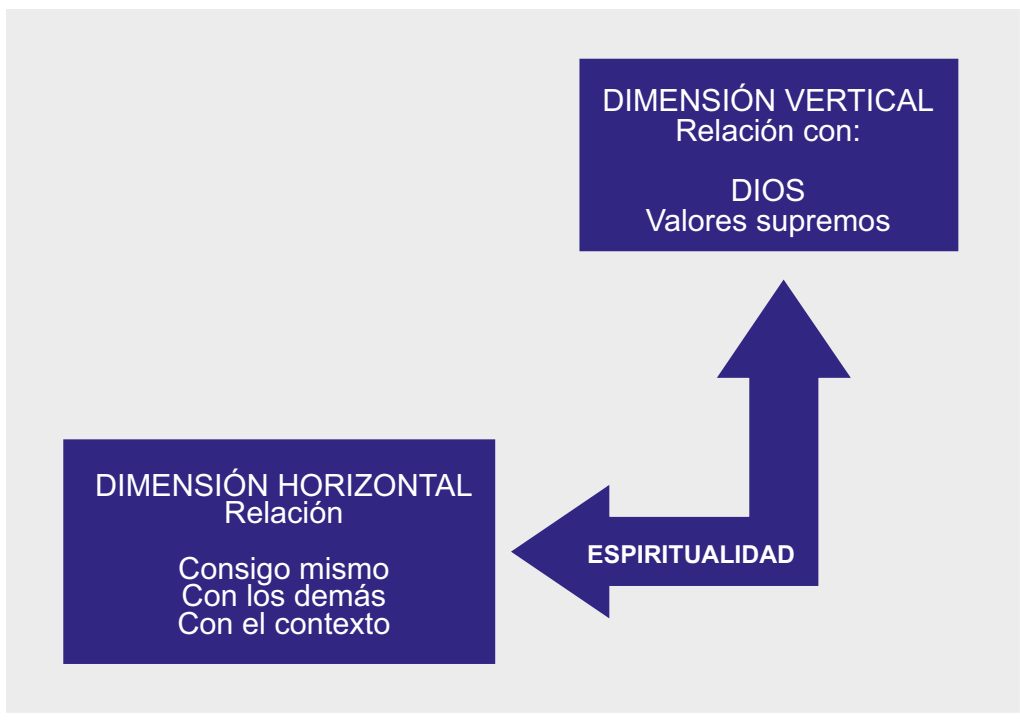

Figura I. Dimensiones de la espiritualidad de "Bienestar espiritual en personas que viven situaciones de enfermedad crónica (pacientes - cuidadores)" por B. Sánchez (2008) modificado por Salgado, 2012.

En relación a las dimensiones que presenta la espiritualidad, Ortiz (2007) considera que las cosas que hacen, sienten y dicen las personas tienen un sentido dentro de su marco existencial y contextual. La espiritualidad no puede desvincularse de las otras condiciones materiales de existencia, ya que se sustenta en las condiciones políticas, sociales, económicas, culturales y biológicas; en que vive y trasciende el ser humano.

\section{Características de la espiritualidad}

Recogiendo diversos aportes, a continuación se presentan las características de la espiritualidad.

$\checkmark$ La espiritualidad al ser entendida como una relación con Dios, provee un significado, propósito y misión de vida.

$\checkmark$ La espiritualidad se puede expresar a través del cuerpo, el pensamiento, los sentimientos, los juicios y la creatividad. Incluye la relación de la persona con los aspectos no materiales de la vida. Agrupa las ideas filosóficas acerca de la vida y su propósito. Tiene el poder de dar forma y significado al ser, saber y hacer. Es un impulso unificador, un sistema de guía interno básico para el bienestar humano. 
No es prerrogativa de los creyentes, puede estar desvinculado de la religión y no ser expresado como una práctica religiosa. Es un componente de la salud relacionado con la esencia de la vida (Sánchez, 2004).

$\checkmark$ La vivencia de la espiritualidad varía de una persona a otra y de un momento a otro de la vida. A su vez, la espiritualidad puede generar bienestar o malestar en las personas (Sánchez, 2009).

$\checkmark$ Muchas veces se solapa o enmascara la verdadera vida espiritual y solo en situaciones extremas de dolor, sufrimiento o pérdida se pone de manifiesto. Por ejemplo, con frecuencia los enfermos que se enfrentan a la muerte manifiestan necesidades de tipo espiritual o trascendente (Bayés, 2006; Murray, 2004; Taylor, 2003; Walter, 1997; como se citó en Rodríguez, 2006).

$\checkmark$ La espiritualidad entendida como la búsqueda de lo sagrado, puede abarcar tanto las expresiones espirituales tradicionales, teocéntricas y de base institucional, como las expresiones no-teístas, apartadas de las creencias y prácticas tradicionales (Pargament \& Mahoney, como se citó en San Martin, 2007) lo cual sería la base para afirmar que la espiritualidad puede ser experimentada por creyentes y no creyentes.

$\checkmark$ La espiritualidad puede ser usada tanto constructiva como destructivamente y su valor depende de la manera específica en que las personas buscan lo sagrado (Pargament \& Mahoney, como se citó en San Martin, 2007).

La espiritualidad puede ser vivida de manera positiva (fe, confianza y abandono en Dios). Bek (como se citó en Ortiz, 2007) señala que la espiritualidad significa cultivar un Dios de amor, de solidaridad y de servicio, no de miedo. Con relación a uno mismo, cultivar la autoestima, y sentido de propósito en la vida; con relación a los demás, cultivar el perdón, la reconciliación, ser inclusivo/a y con relación a la naturaleza, el cultivo del respeto a la creación, su cuidado y disfrute en acción de gracias. La espiritualidad produce unos estados afectivos, tales como el altruismo, amor, caridad, misericordia y el perdón, que tienen un efecto significativo en las relaciones de la persona consigo mismo, con los demás y con Dios.

$\checkmark$ Las variables relativas a espiritualidad tienden a acompasarse a un rango de conceptos como significado, totalidad, trascendencia, conexión, gozo y paz, y no requieren de la participación en una organización religiosa (Martínez et al., 2004).

La espiritualidad tiende a asociarse con la trascendencia, con la esperanza, propósito y sentido (Frankl, 1988, 1999a; Flannelly, Weaver \& Costa, 2004) o con 
aspectos inmateriales de la existencia (Meraviglia, 2004). La espiritualidad apunta según Musick et al. ( 1989) a la trascendencia o a la búsqueda de algo más grande que uno mismo. La espiritualidad representa la búsqueda de significado existencial (Monge \& León, 1999). De acuerdo a Bayés (2006), Fitchett \& Handzo (1989), Frankl (1990a, 1999), Musick et al. (1989) la espiritualidad puede proporcionar una sensación de significado y propósito (como se citó en Rodríguez, 2006).

$\checkmark$ La espiritualidad conlleva la creencia en algún significado o algún orden en el universo, significa aceptación de lo que es (no resignación), y aptitud para hallar la paz y la felicidad en un mundo imperfecto, lo que genera creatividad y capacidad de amar desinteresadamente (Siegel, como se citó en Rodríguez, 2006).

$\checkmark$ La espiritualidad implica un sistema de creencias acerca del sentido de la vida, de la muerte, de la enfermedad y otros temas existenciales (Ellison, 1991; Reed, 1991; Taylor, 200I, como se citó en Rodríguez, 2006).

De acuerdo a Pinto (2007) la espiritualidad puede existir por niveles; mientras más alto el nivel de ella, mayor la influencia sobre la vida y la salud. Aunque la fuerza vital inherente, que es el espíritu de una persona, no se debilita por sí mismo, el reflejo de espiritualidad de una persona puede ser afectado por los factores físicos o ambientales que influencian la armonía de la vida.

A continuación, se presenta la figura 2, que muestra cuáles serían algunas de las principales características de la espiritualidad, a través de las cuales se puede apreciar que la base de todo, es la relación que se establece con Dios, que provee un significado, propósito y misión en la vida. 

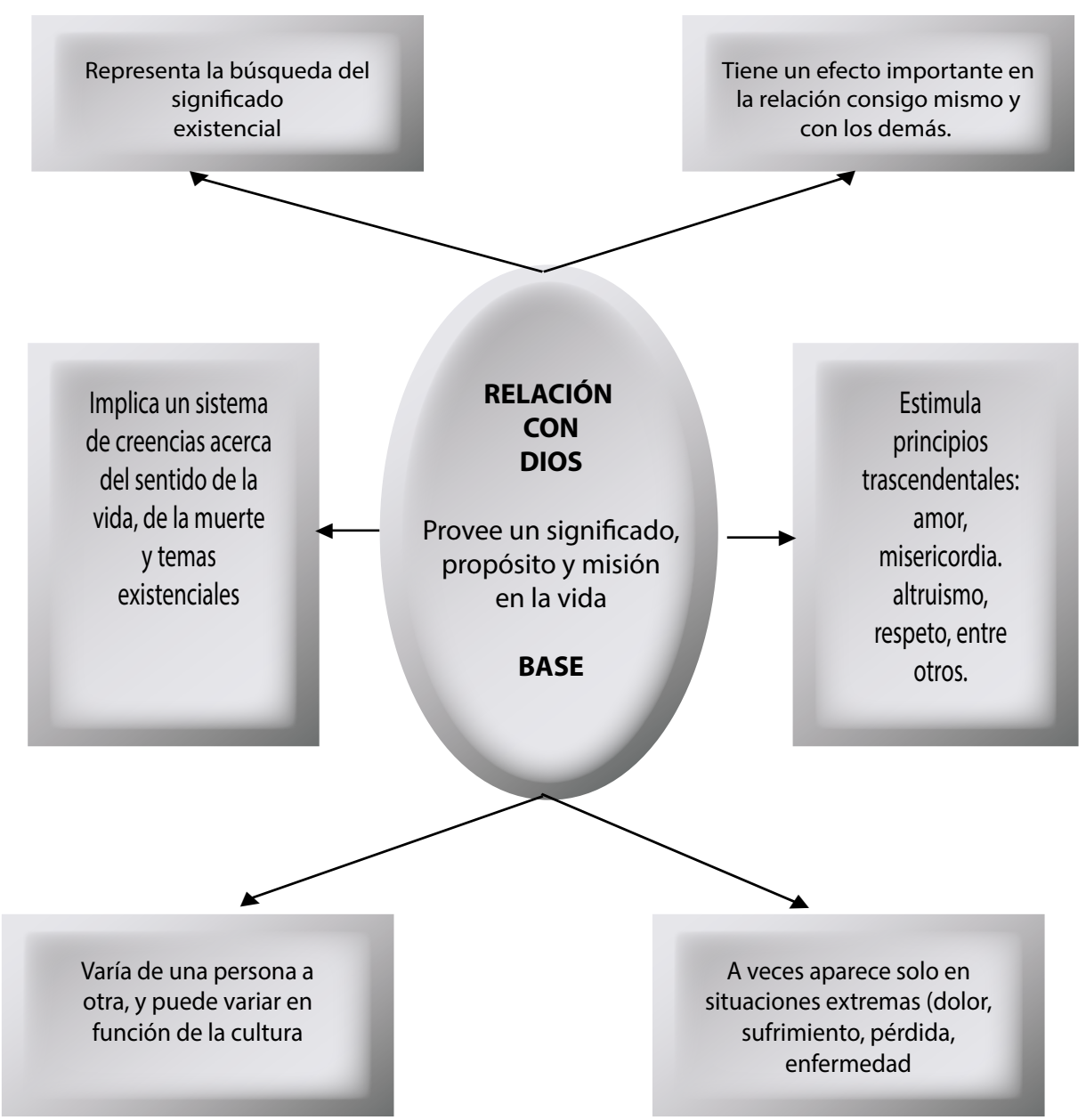

Figura 2. Características principales de la espiritualidad de "Efectos del bienestar espiritual sobre la resiliencia en estudiantes universitarios de Argentina, Bolivia, Perú y República Dominicana" por C.Salgado-Lévano, 2012. 


\section{A MODO DE CONCLUSIÓN}

Existe un serio interés en la comunidad científica por abordar variables íntimamente ligadas a la vida del ser humano como son, el afrontamiento religioso, la convicción espiritual, el bienestar espiritual, las experiencias y formas de participación religiosa, creencias y prácticas espirituales y religiosas, la conversión y la fe en Dios, entre otras; lo cual se ha traducido en importantes investigaciones que se encuentran en libros, revistas científicas y son punto de discusión en congresos internacionales; sin embargo, y a pesar de ser un área muy explorada en otras realidades (vg. norteamericana, europea) es algo que en países latinoamericanos, no se ha investigado de manera satisfactoria, antes bien, se ha descuidado.

En esta línea, si bien es cierto aún existen muchas barreras y cierto oscurantismo en algunos sectores académicos; es imprescindible sumar esfuerzos personales y colectivos para impulsar investigaciones vinculadas al campo de la Psicología de la Religión y Espiritualidad, dado el innegable rol que cumplen en la vida del ser humano, para darle sentido, una motivación para seguir, la posibilidad de trascender y de estar en armonía consigo mismo, con los demás y especialmente con Dios. 


\section{REFERENCIAS}

Beca, J. (2008). El cuidado espiritual del enfermo como responsabilidad del profesional de la salud. Ética de los Cuidados, I (I). Recuperado de http://www.index-f. com/eticuidado/nl/et6734.php

Gallego-Pérez, J., García-Alandete, J. y Pérez-Delgado, E. (2007). Factores del Test purpose in life y religiosidad. Univ. Psychol, 6 (2), 213-229.

García Alandete, J. (2002). Actitudes religiosas, valores y razonamiento moral. Tesis Doctoral. Universitat de Valencia. Facultat de Psicologia. Departament de Psicologia Bàsica. Valencia.

García-Alandete, J. y Pérez, E. (2005). Actitudes religiosas y valores en un grupo de jóvenes universitarios españoles. Anales de Psicología, 2 I (I), I49- 169.

González, T. (2004). Las creencias religiosas y su relación con el proceso saludenfermedad. Revista Electrónica de Psicología Iztacala, 7 (2), 19-29. Recuperado de www.iztacala.unam.mx/carreras/psicologia/psiclin/.../vol7no2art2.pdf

Jaramillo, A., Carvajal, S. Marín, N. y Ramírez, A. (2008). Los estudiantes universitarios Javerianos y su respuesta al sentido de la vida. Pensamiento Psicológico, 4 ( I I), 199-208.

Jiménez, J. (2005). La espiritualidad, dimensión olvidada de la Medicina. Revista Gaceta Universitaria, I, 92-101.

Korman, G., Garay, C. y Sarudiansky, M. (2008). Psicoterapia cognitiva y religión ¿Qué debe saber un terapeuta cognitivo de la diversidad religiosa de la Capital Federal y el Conourbano Bonaerense? Summa Psicológica UST. 5 (2), 53-62.

Martínez, Ma. (2006). El estudio científico de las fortalezas trascendentales desde la Psicología Positiva. Clínica y Salud, 17 (3), 245-258.

Martínez, M., Méndez, C. y Ballesteros, B. (2004). Características espirituales y religiosas de pacientes con cáncer que asisten al Centro Javeriano de Oncología. Univ. Psychol. 3 (2), 23I-246.

Montero, M. y Sierra, L. (1996). Escala de Bienestar Espiritual: un estudio de validación. La Psicología Social en México, AMEPSO, VI, 28-33.

Ortiz, M. (8 de Agosto del 2007). Dilemas Éticos de la Espiritualidad en Trabajo Social. Conferencia ofrecida en la Quinta Conferencia de Trabajo Social Forense, Universidad interamericana, Recinto Metropolitano. Puerto Rico. 
Pérez Santiago, J.A. (2007). Estudio exploratorio sobre el tema de la espiritualidad en el ambiente laboral. Anales de Psicología, 23 (I), I37- 46.

Pinto, N. (2007). Bienestar espiritual de los cuidadores familiares de niños que viven enfermedad crónica, Investigación en Enfermería: Imagen y Desarrollo, 9 (I), 20-35.

Quiceno, J. y Vinaccia, S. (2009). La salud en el marco de la psicología de la religión y la espiritualidad Diversitas. Perspectivas en Psicología, 5 (2), 32 I-336.

Quiceno, J. y Vinaccia, S. (20II). Creencias-Prácticas y Afrontamiento espiritualreligioso y características sociodemográficas en enfermos crónicos. Psychologia: Avances de la disciplina, 5 (I), 25-36.

Rivera, A. (2007). Modelo de intervención racional emotivo para la promoción del ajuste psicológico en el adulto mayor en un contexto religioso. (Tesis doctoral inédita). Universidad Nacional Autónoma de México.

Rodríguez, Ma. (2006). Afrontamiento del cáncery Sentido de la vida: un estudio empírico y clínico. (Tesis doctoral, Universidad Autónoma de Madrid, España). Recuperada de https://repositorio.uam.es/bitstream/handle/I0486/25 I6/I49| rodriguez_fernandez_maria_isabel.pdf?sequence $=$ I

Rodríguez, J. (2007). La religión como predictor de las actitudes hacia la nación. Actualidades en Psicología, 21, 167-191.

Rodríguez, M., Fernández, M.L., Pérez, M.L. y Noriega, R. (20l I). Espiritualidad variable asociada a la resiliencia. Cuadernos Hispanoamericanos de Psicología, I (2), 24-49.

Salgado, C. (2012). Efectos del bienestar espiritual sobre la resiliencia en estudiantes universitarios de Argentina, Bolivia, Perú y República Dominicana. (Tesis doctoral, Universidad Nacional Mayor de San Marcos, Lima, Perú). Recuperada de http://cybertesis.unmsm.edu.pe/bitstream/cybertesis/3293/I/Salgado_la.pdf

Sánchez, B. (2004). Dimensión espiritual del cuidado de Enfermería en situaciones de cronicidad y muerte. Bogotá: Universidad Nacional de Colombia.

Sánchez, G. (2007). Unapsicologíapara una espiritualidad. Catholic.netInc. Recuperado de www.es.catholic.net/religiosas/805/I353/articulo.php?id=35368

Sánchez, B. (2008). Bienestar espiritual en personas que viven situaciones de enfermedad crónica (pacientes - cuidadores). Universidad Nacional de Colombia. Facultad de enfermería. Recuperado de www.gcronico.unal.edu. co/.../ESPIRITUALIDAD\%20SEM.pdf 
Sánchez, B. (2009). Bienestar espiritual en personas con y sin discapacidad. Aquichan, $9(\mathrm{I}), 8-22$.

San Martin, C. (2007). Espiritualidad en la tercera edad. Psicodebate 8. Psicología, Cultura y Sociedad. pp. II I-128.

Vanistendael, S. (2003). Resiliencia y espiritualidad. El realismo de la fe. Ginebra, Suiza: Oficina Internacional Católica de la Infancia. 